it was anticipated that a stream of slow neutrons would diffuse more readily along an air column eontained in a tube having walls composed of a substance of high hydrogen content, than through the walls themselves.

We have verified Dr. Szilard's prediction in the following manner. Neutron sources consisting either of about 100 millicuries of radon with beryllium, or of 150 milligrams of radium in tubes surrounded by beryllium, were embedded in paraffin blocks $10 \mathrm{~cm}$. in diameter and $10 \mathrm{~cm}$. high, and placed inside a large cavity in the base of a hollow cylinder of paraffin wax. The walls of the cylinder were $7-10 \mathrm{~cm}$. thick, and its length could be altered by the addition of a number of similar sections of $12 \mathrm{~cm}$. internal diameter. The intensity of the neutron beam at various distances from the source was measured by the radioactivity induced in thin pieces of silver or rhodium or in iodine; the activity of the detectors being measured by a Geiger-Müller counter.

In this way, appreciable radioactivation was obtained at distances exceeding one metre from the source. A right angle bend in the tube reduced the intensity of the neutron beam by about 30 per cent. To obtain the maximum canalising effect, it was found necessary to avoid all gaps in the walls and to close both ends of the canal with wax. A tube of pure graphite gave a smaller but definite canalising effect, but none was observed with a tube of adulterated ebonite.

Fuller details of these experiments will appear in a forthcoming issue of the British Journal of Radiology, and other observations will be published inde. pendently by Dr. Szilard.

F. L. Hopwood.

T. A. Chalmers.

St. Bartholomew's Hospital Medical College, London. Feb. 19.

${ }^{1}$ La Ricerca Scientifica, V, 2, 7-8; 1934. V, 2, 380; 1934. VI, 1,$2 ; 1935$

\section{The Absolute Field Constant in the New Field Theory}

Is the modification of Maxwell's theory proposed by one of us', the notion of an 'absolute field', called $b$, played an essential part. In the electrostatic case, the universal constant $b$ is simply the upper limit of the field strength, whilst in the general case of an arbitrary field, $b$ sets a limit to the possible values of $\sqrt{ }\left(\vec{E}^{2}-\vec{H}^{2}\right)$, when $\vec{E}$ and $\vec{H}$ are calculated in that Lorentz frame in which the Poynting vector vanishes in the given world-point. (In the exceptional case when there is no such Lorentz frame, that is,

if $\vec{E}$ is perpendicular to $\vec{H}$ and $\vec{E}^{2}=\vec{H}^{2}$, there is no limit.) Born and Infeld ${ }^{2}$ have calculated $b$ from the experimental values of the charge $e$ and mass $m$ of the electron by equating to $m c^{2}$ the total energy of that centrally symmetrical electrostatic solution which has the total charge $e$. By this procedure $b$ works out to be $9 \cdot 18 \times 10^{15}$ E.S.U.

We now believe that this determination may be wrong, notably too high, because the spin had been neglected. Since the solution for the spinning electron cannot yet be calculated, we must content ourselves with giving a rough estimate. Let $\mu$ be the magnetic moment of the spin and $r^{\prime}$ the new radius of the electron (to be calculated here) and let us try tentatively to account for the observed mass $m$ by the energy of the spin only (neglecting the electrostatic energy). The following statement will then be correct as to order of magnitude :

$$
\frac{1}{2} \frac{\mu^{2}}{r^{\prime 3}}=m c^{2} \text {. }
$$

We can assume that $\mu$ is Bohr's magneton :

$$
\mu=\frac{e h}{4 \pi m c^{2}}=\frac{e}{2} \cdot \frac{e^{2}}{m c^{2}} \cdot \frac{h c}{2 \pi e^{2}}=\frac{e r_{0}}{2 \alpha},
$$

where

$$
\alpha=\frac{h c}{2 \pi e^{2}}=\frac{1}{137 \cdot 2},
$$

the fine-structure constant, and $r_{0}=e^{2} / m c^{2}$, the quantity usually called the radius of the electron; it is connected with the electronic radius $r_{e l}$ of the new field theory by the equation

$$
r_{e l}=1 \cdot 236 r_{0} \text {. }
$$

From our first equation we find now :

$$
r^{\prime}=\frac{r_{0}}{2 \alpha^{2 / 3}}=\frac{r_{e l}}{2 \cdot 472 \times \alpha^{2 / 3}}=11 r_{e l} .
$$

Since $r^{\prime}$ is considerably larger than $r_{e l}$, the electrostatic energy in the new model will be a small fraction of $m c^{2}$, and we are justified in neglecting it for our rough estimate.

Again, the field 'in the interior' of the magneton may safely be equated to the absolute field $b$, which fact, as to the order of magnitude, will be expressed by

$$
b=\frac{\mu}{r^{\prime 3}}=\frac{2 m c^{2}}{\mu}=4 \alpha \cdot \frac{e}{r_{0}} \cdot \frac{m c^{2}}{e^{2}}=4 \alpha \cdot \frac{e}{r_{0}^{2}} .
$$

This is to be compared with the value, say $b_{e l}$, formerly calculated from the electrostatic energy :

We have

$$
b_{e l}=\frac{e}{r_{e l^{2}}}=\frac{e}{\left(1 \cdot 236 r_{0}\right)^{2}} .
$$

$$
b=4(1 \cdot 236)^{2} \cdot \alpha \cdot b_{e l}=\frac{b_{e l}}{22 \cdot 5} .
$$

If the estimates are not too rough, the new point of view increases the radius of the electron by a factor of about 10 , and decreases the limiting field to about the twentieth part.

Cambridge.

Oxford. Jan. 28.

$$
\text { MAX BorN. }
$$

ERWIN SchröDINGER.

${ }^{1}$ M. Born, NATURR, 132, $282 ; 1933$.

${ }^{1}$ M. Born, NATURE, 132, $282 ; 1933$.
${ }^{2}$ M. Born and L. Infeld, Proc. Roy. Soc., A, 144, 426 ; 1934.

\section{The Hypothesis of Continental Drift}

The late Dr. A. Wegener claimed the advantage for his hypothesis of 'continental drift' that it could be tested by making repeated astronomical observations of the positions of land stations; for drifts at the rates of only a few feet a year would suffice to carry the land masses far within the span of geological time. Few geologists would in respect to this problem deny the applicability of the dictum that 'the present is the key to the past'; the question is whether precision determinations of 'position' will give measurable results within a reasonable time.

In 1932 Herr Hans Jelstrup determined the 